\title{
Effects of cadmium and zinc toxicity on orientation behaviour of Echinoparyphium recurvatum (Digenea: Echinostomatidae) cercariae
}

\author{
N. J. Morley*, M. Crane, J. W. Lewis \\ School of Biological Sciences, Royal Holloway, University of London, Egham, Surrey TW20 0EX, United Kingdom
}

\begin{abstract}
The effects of cadmium and zinc toxicity on orientation behaviour (photo- and geo-taxis) of Echinoparyphium recurvatum cercariae was investigated at concentrations ranging from 10 to $1000 \mu \mathrm{g} \mathrm{l}^{-1}$. Exposure to the toxicants at all metal concentrations caused a change in orientation to negative phototaxis and positive geotaxis during the submaximal dispersal phase $(0.5 \mathrm{~h}$ cercarial age $)$. Autometallography staining of cercariae exposed to $1000 \mathrm{\mu g} \mathrm{l}^{-1}$ cadmium or zinc showed selective binding of heavy metals to tegumental surface sites associated with sensory receptors. The significance to parasite transmission of changes in cercarial orientation behaviour in metal polluted environments is discussed.
\end{abstract}

KEY WORDS: Echinoparyphium recurvatum - Cercariae · Phototaxis · Geotaxis · Autometallography · Cadmium · Zinc

Resale or republication not permitted without written consent of the publisher

The swimming behaviour of cercariae ensures optimal departure from the habitat of the first intermediate host, dispersal, arrival and maintenance within the environment of the next host (Haas 1994). In species of cercariae that infect snails as second intermediate hosts, there is a particular advantage in leaving the habitat of the shedding snail host to prevent superinfection of the first intermediate host. Haas (1994), McCarthy (1999) and Loy et al. (2001) have demonstrated that several species of echinostome cercariae have a dispersal phase followed by a host-location phase which involves changes in cercarial orientation towards light and gravity. In particular McCarthy (1999) demonstrated that a change in phototactic orientation of Echinoparyphium recurvatum cercariae coincides with an initial phase of sub-maximal infectivity during dispersal (positive phototaxis) leading to a subsequent phase of maximal infectivity during host-location (negative phototaxis).

Heavy metal pollution may have an effect on dispersal and host-location behaviour that could influence transmission from one host to another, especially at metal concentrations that have only slight overall toxic effects on cercarial infectivity. Morley et al. (2001) demonstrated an avoidance behaviour in Schistosoma mansoni miracidia exposed to cadmium and zinc. Indeed, the heavy metal stain silver nitrate has been shown to severely inhibit chemo-orientation of echinostome cercariae towards their second intermediate host (Korner \& Haas 1998). Historically, silver nitrate is mainly used to highlight areas on the cercarial body surface associated with sensory receptors because it selectively binds to these structures (Richard 1971). Such selective surface-binding may also be replicated by other heavy metal pollutants, of which the mechanisms of cercarial uptake are unknown.

The aims of the present study were (1) to investigate the effect of cadmium and zinc toxicity on the phototactic and geotactic responses of Echinoparyphium recurvatum cercariae during phases of sub-maximal and maximal infectivity, and (2) using the autometallography staining procedure devised by Danscher (1984) and Danscher et al. (1987), to establish if any metal-induced changes in orientation behaviour might be due to these metals selectively binding to areas associated with sensory receptors. Cadmium and zinc are related heavy metals which almost always occur together in pollution incidents. In the UK, chronic pollution of the aquatic environment has been recorded at concentrations as high as $160 \mu \mathrm{g} \mathrm{l}^{-1}$ for $\mathrm{Cd}$ and $8800 \mu \mathrm{g}$ $\mathrm{l}^{-1}$ for Zn (Vivian \& Massie 1977).

Materials and methods. Preparation of stock and test solutions and the analysis of metal loss from the test solutions were as previously described by Morley et al. (2002a).

Phototaxis was investigated using a simple light/ dark choice chamber constructed from a small, clear, circular glass Petri dish (6 cm in diameter, $1.5 \mathrm{~cm}$ in depth), half of which was blacked out using light-proof black PVC tape. In each replicate experiment, a smaller polystyrene Petri dish ( $5 \mathrm{~cm}$ in diameter, $1 \mathrm{~cm}$ in depth) was placed inside the choice chamber. Experiments were carried out in a darkened room maintained at a constant 
temperature of $20^{\circ} \mathrm{C}$. The choice chamber was illuminated directly from above by a cold-light source (light intensity 500 lux). The polystyrene Petri dish was filled with $20 \mathrm{ml}$ of synthetic hard water $\left(250 \mathrm{mg} \mathrm{l}^{-1} \mathrm{CaCO}_{3}\right.$, pH 8.00) (HMSO 1969) containing either a 10, 100, or $1000 \mu \mathrm{g} \mathrm{l}^{-1}$ metal-ion concentration of cadmium, zinc, cadmium/zinc mixture or distilled water (control). We introduced 20 Echinoparyphium recurvatum cercariae (maximum age $10 \mathrm{~min}$ ) shed from Lymnaea peregra collected from Bushy Park, Greater London (National Grid Reference TQ160694), and identified according to Nasir (1984), into the centre of the choice chamber. The cercariae were allowed to disperse for $20 \mathrm{~min}$, and their distribution was then determined, i.e. at $0.5 \mathrm{~h}$ cercarial age, which is regarded as the sub-maximal infectivity phase (McCarthy 1999). A subsequent reading was taken after $2 \mathrm{~h}$, i.e. $2.5 \mathrm{~h}$ cercarial age, which is regarded as the maximal infectivity phase (McCarthy 1999). The experiment was repeated 10 times, using a new Petri dish each time. Data was arcsine-transformed and analysed with the SPSS computer package using Tukey's HSD test.

Geotaxis was investigated with a long-armed stereo microscope set up to provide a near-horizontal view back onto the microscope stand. A $4.5 \mathrm{ml}$ disposable polystyrene cuvette (Greiner Laboratory Supplies) was placed on the microscope stand with a marker-pen line dividing it equally into 2 geotactic zones. The experiment was undertaken in a darkened room at $20^{\circ} \mathrm{C}$; the cuvette was illuminated with a cold-light source (light intensity 500 lux) from the two sides, positioned $20 \mathrm{~cm}$ from the cuvette to provide an evenly distributed light source which would not interfere with the geotactic readings by inducing any overriding phototactic

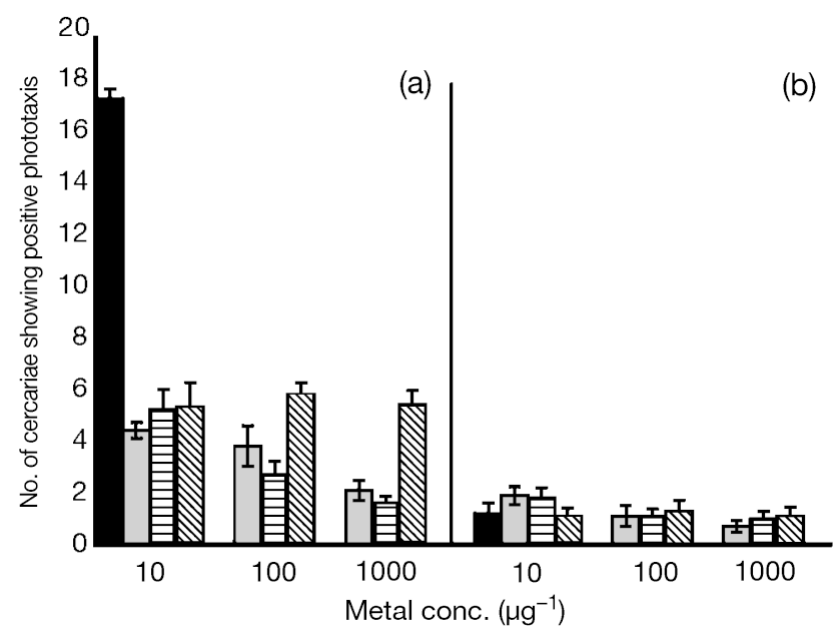

Fig. 1. Echinoparyphium recurvatum. Toxic effects of heavy metals on phototactic behaviour of cercariae at age of (a) $0.5 \mathrm{~h}$ and (b) $2.5 \mathrm{~h}$. Data show number of cercariae displaying positive phototaxis $(\mathrm{n}=20)$; error bars $= \pm \mathrm{SE}_{\text {; }}(\mathbf{\square})$ control;

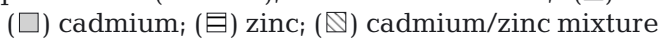

behaviour. The cuvette was filled with $4 \mathrm{ml}$ of synthetic hard water (HMSO 1969) containing either 10, 100, or $1000 \mathrm{~g} \mathrm{l}^{-1}$ of cadmium, zinc, cadmium/zinc mixture, or distilled water (control). We introduced 20 Echinoparyphium recurvatum cercariae (maximum age $10 \mathrm{~min}$ ) into the solution and allowed them to disperse for 20 min (cercarial age $0.5 \mathrm{~h}$ ). The distribution of the cercariae was then counted. Cercariae above the markerpen line were regarded as negatively geotactic, those below the line as positively geotactic. A subsequent reading was taken after $2 \mathrm{~h}$ cercarial distribution, i.e. at cercarial age $2.5 \mathrm{~h}$. The experiment was repeated 10 times, using a new cuvette each time. Data was arcsine-transformed and analysed with the SPSS computer package using Tukey's HSD test.

For autometallography staining of cercariae we employed the staining technique of Danscher et al. (1987). This utilises a simple silver-enhancement kit, normally used to induce the precipitation of metallic silver onto the surface of colloidal gold particles for signal amplification in immunocytochemistry. In the present study the technique was modified for whole mounts and employed the improved type of silver-enhancer kit (Sigma Chemicals) currently available. Cercariae were exposed to $1000 \mu \mathrm{g}$ $\mathrm{l}^{-1} \mathrm{Cd}$ or Zn for $30 \mathrm{~min}$ in hard water (HMSO 1969) and then fixed in $70 \%$ alcohol (controls were exposed to hard water for only $30 \mathrm{~min}$ ). Cercariae were subsequently rehydrated to distilled water, stained for 6 min using the silver-enhancer kit, rinsed in distilled water, fixed in $2.5 \%$ sodium thiosulphate for $4 \mathrm{~min}$, rinsed again in distilled water, then dehydrated, cleared in histoclear and mounted on glass slides. Metals were visualised as black deposits on the cercarial surface.

Water analysis revealed that only a small amount of dissolved metal loss occurred in the test solutions and only at the highest test concentrations $\left(1000 \mu \mathrm{g} \mathrm{l}^{-1}\right)$; this did not exceed more than $5 \%$ over the exposure period.

Results. The effect of all 3 metal solutions on cercarial phototaxis at all concentrations was to cause a change at $0.5 \mathrm{~h}$ from a predominantly positive phototaxis to a predominantly negative phototaxis (Fig. 1). The change in phototactic orientation was significantly different in all test solutions compared to controls (Tukey's HSD test, $\mathrm{p}$ $<0.001$ ). There was no significant difference between cadmium- and zinc-exposed cercariae (Tukey's test $\mathrm{p}=$ 0.961), but $\mathrm{Cd} / \mathrm{Zn}$ mixture effects were significantly different from both single-metal-exposed cercariae (Tukey's test $\mathrm{p}=0.001$ ). After $2.5 \mathrm{~h}$ there was no difference between any of the exposures, with all cercariae, including controls, demonstrating predominantly negative phototaxis (Fig. 1).

Cercarial geotaxis in controls changed from predominantly negative geotaxis $(0.5 \mathrm{~h})$ to predominantly positive geotaxis (2.5 h: Fig. 2), whereas the metal solu- 


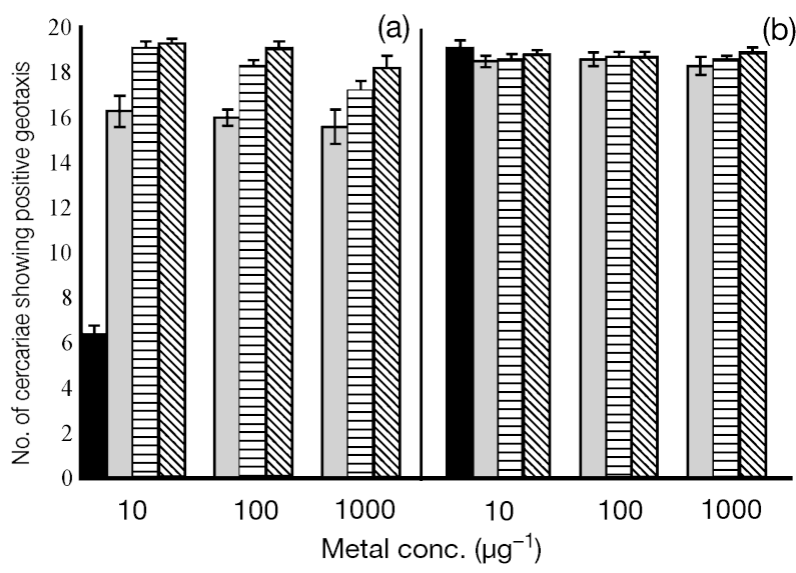

Fig. 2. Echinoparyphium recurvatum. Toxic effects of heavy metals on geotactic behaviour at age of (a) $0.5 \mathrm{~h}$ and (b) $2.5 \mathrm{~h}$. Data show number of cercariae displaying positive geotaxis $(\mathrm{n}=20)$; error bars $= \pm \mathrm{SE}_{\text {; }}(\square)$ control; $(\square)$ cadmium; $($ 目) zinc;

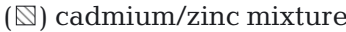

tions induced a change from predominantly negative geotaxis to positive geotaxis at $0.5 \mathrm{~h}$. This change in geotaxis was significant in all test solutions compared to controls (Tukey's test $\mathrm{p}<0.001$ ). There was a significant difference between cadmium and zinc exposures (Tukey's test $\mathrm{p}<0.001$ ); however, $\mathrm{Cd} / \mathrm{Zn}$ mixture effects were only significantly different from those of cadmium exposed cercariae (Tukey's test $\mathrm{p}<0.001$ ). After $2.5 \mathrm{~h}$ there was no difference between any of the exposures, with all cercariae demonstrating positive geotaxis (Fig. 2).

Autometallography staining of metal-exposed cercariae demonstrated selective binding of metals to the cercarial surface. There was no apparent difference between cadmium and zinc in the areas stained, although the resolution was poor in both cases, with zinc being slightly better than cadmium in this respect. Control cercariae showed no metal staining on any part of their surface; however, they did demonstrate some weak staining of the primary excretory duct which was not replicated in metal-exposed cercariae. Dense staining of metal-exposed cercariae occurred on the cephalic region and posterior tip of the tail (Fig. 3). The cephalic region of the cercarial body contained both dense stains around and on the mouth and smaller pinpoint stains (Fig. 3b). In addition, pin-point staining occurred across the cercarial body surface (Fig.3a).

Discussion. The present study demonstrated that metals can have a significant impact on the orientation behaviour of Echinoparyphium recurvatum cercariae. The change in orientation of control cercariae from 0.5 to 2.5 $\mathrm{h}$ is in accordance with previous observations of positive to negative changes in phototaxis in E. recurvatum cercariae (McCarthy 1999), and a geotactic change in Pla- giorchis elegans cercariae from negative to positive (Lowenberger \& Rau 1994). The nature of metal toxicity demonstrated here may be either a reflex response to the toxic environment or a by-product of the toxic action of metals on the cercariae.In both cases, it is possible that changes in normal orientation behaviour may be associated with the selective binding of metals to areas on the tegument surface which broadly correlate with a number of sensory receptor sites, especially in the cephalic region mapped out by Grabda-Kazubska \& Kiseliene (1989) using silver nitrate stain. Nevertheless, the distribution pattern of metal-binding in the present study may also reflect binding to other surface structures, in particular glandular openings in the cephalic region, excretory pores and the mouth opening, all of which can be stained with silver nitrate (Short \& Cartrett 1973). How-

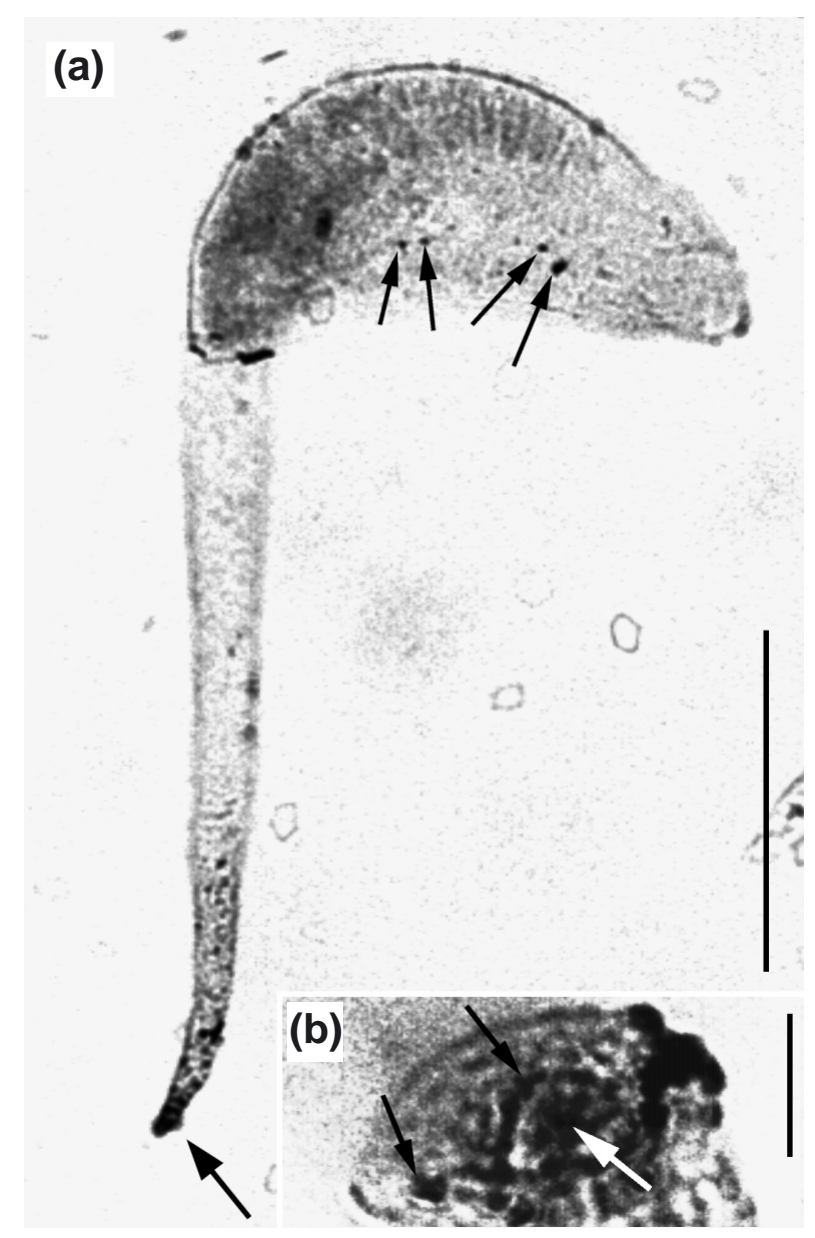

Fig. 3. Echinoparyphium recurvatum. Autometallography staining of cercariae exposed to $1000 \mu \mathrm{g} \mathrm{l}^{-1} \mathrm{Zn}$ for $30 \mathrm{~min}$. (a) Whole cercariae; note staining along body length (thin arrows) and heavy staining at tail tip (thick arrow); scale bar = $200 \mu \mathrm{m}$. (b) Cephalic region, note pinpoint staining around mouth (black arrows) and staining of mouth itself (white arrow); scale bar $=50 \mu \mathrm{m}$ 
ever, it appears that not all sensory receptors described by Grabda-Kazubska \& Kiseliene (1989) selectively bind the metals. This may reflect the different types of sensory endings present on cercariae (Zdarska 1992, Czubaj \& Niewiadomska 1996), and since structurally different receptors are regarded as having different functions (Czubaj \& Niewiadomska 1996), it suggests that possibly only a limited amount of sensory impairment results from metal toxicity.

Alternatively, changes in cercarial behaviour may be a reaction to unfavourable environmental conditions consisting of reducing the phase of normal distribution behaviour and attempting to make contact with a suitable snail host more rapidly. Studies by Korner \& Haas (1998) on the effects of silver nitrate on the chemo-orientation of Pseudochinoparyphium echinatum and Echinostoma revolutum showed that exposed cercariae, if washed extensively 3 times with dechlorinated tap water, largely regained their chemo-orientation abilities, suggesting that metal toxicity did not permanently inhibit cercarial behaviour. Indeed, in the present study, toxicantinduced changes in the chemo-orientation of Echinoparyphium recurvatum cercariae might also have been occurring, although this was not determined. Other studies have noted that Notocotylus attenuatus also alters its encystment behaviour when exposed to heavy metals (Morley et al. 2002b), and an increased number of E. recurvatum cercariae become encysted within the first intermediate host on exposure to cadmium (N. J. Morley unpubl. obs.). This may be indicative of a behavioural change in response to unfavourable environmental conditions in these cercarial species.

Whatever the mechanism of toxic action, the most important consideration is whether changes in orientation are accompanied by changes in infectivity. Morley et al. (2002a) demonstrated that Echinoparyphium recurvatum cercariae exposed to cadmium and zinc for a short duration (30 min) exhibited reduced transmission when exposed to concentrations similar to those used in the present study. McCarthy (1999) and the present study demonstrated that under control conditions the initial positive phototaxis and negative geotaxis of E. recurvatum cercariae are coincident with the initial sub-maximal infectivity phase described by Evans \& Gordon (1983). If a change in infectivity coincides with the behavioural changes recorded in the present study, this would probably indicate that cercariae are capable of responding to unfavourable environmental conditions in order to ensure transmission success and maintain the life cycle of E. recurvatum. An investigation of these potential behaviour/infectivity interactions would provide a fundamental basis for future study.

Editorial responsibility: Thomas Braunbeck, Heidelberg, Germany

\section{LITERATURE CITED}

Czubaj A, Niewiadomska K (1996) Ultrastructure of sensory endings in Diplostomum pseudospathaceum Niewiadomska, 1984 cercariae (Digenea, Diplostomidae). Int J Parasitol 26:1217-1225

Danscher G (1984) Autometallography. A new technique for light and electron microscopic visulization of metals in biological tissues (gold, silver, metal sulphides and metal selenides). Histochemistry 81:331-335

Danscher G, Rytter Norgaard JO, Baatrup E (1987) Autometallography: tissue metals demonstrated by a silver enhancement kit. Histochemistry 86:465-469

Grabda-Kazubska B, Kiseliene V (1989) Chaetotaxy and excretory system of the cercariae of Echinoparyphium recurvatum (Linstow, 1873) (Digenea, Echinostomatidae). Acta Parasitol Pol 34:325-335

Evans NA, Gordon DM (1983) Experimental studies on the transmission dynamics of the cercariae of Echinoparyphium recurvatum (Digenea: Echinostomatidae). Parasitology 87:167-174

Haas W (1994) Physiological analysis of host-finding behaviour in trematode cercariae: adaptations for transmission success. Parasitology (Suppl) 109:S15-S29

HMSO (Her Majesty's Stationery Office) (1969) Fish toxicity tests. HMSO Leafl, No. Dd. 139779 K36 12/69, HMSO Publications Centre, London

Korner M, Haas W (1998) Chemo-orientation of echinostome cercariae towards their snail hosts: amino acids signal a low host-specificity. Int J Parasitol 28:511-516

Lowenberger CA, Rau ME (1994) Plagiorchis elegans: emergence, longevity and infectivity of cercariae, and host behavioural modifications during cercarial emergence. Parasitology 109:65-72

Loy C, Motzel W, Haas W (2001) Photo- and geo-orientation by echinostome cercariae results in habitat selection. J Parasitol 87:505-509

McCarthy AM (1999) Phototactic responses of the cercariae of Echinoparyphium recurvatum during phases of sub-maximal and maximal infectivity. J Helminthol 73:63-65

Morley NJ, Crane M, Lewis JW (2001) Toxicity of cadmium and zinc to miracidia of Schistosoma mansoni. Parasitology 122:81-85

Morley NJ, Crane M, Lewis JW (2002a) Toxic effects of cadmium and zinc on the transmission of Echinoparyphium recurvatum cercariae. J Helminthol 76:157-163

Morley NJ, Crane M, Lewis JW (2002b) Toxicity of cadmium and zinc to encystment of Notocotylus attenuatus (Trematoda: Notocotylidae) cercariae. Ecotoxicol Environ Saf 53:129-133

Nasir P (1984) British freshwater cercariae. Universidad de Oriente Press, Cumana, Venezuela

Richard J (1971) La chetotaxie des cercaries. Valeur systematique et phyletique. Mem Mus Natl Hist Nat Ser A Zool 67:1-179

Short RB, Cartrett ML (1973) Argentophilic 'papillae' of Schistosoma mansoni cercariae. J Parasitol 59:1041-1059

Vivian CMG, Massie KS (1977) Trace metals in waters and sediments of the River Tawe, South Wales, in relation to local sources. Environ Pollut 14:47-61

Zdarska Z (1992) Transmission electron microscopy of sensory receptors of Echinostoma revolutum (Froelich 1802) cercariae (Digenea: Echinostomatidae). Parasitol Res 78: $598-606$

Submitted: August 7, 2002; Accepted: March 19, 2003

Proofs received from author(s): July 14, 2003 